\title{
Fiber MOPAs with high control and high power
}

\author{
J. Nilsson, S. Yoo, P. Dupriez*, C. Farrell, M. S. Z. Abidin, J. Ji, J.-N. Maran, C. A. Codemard, \\ Y. Jeong, J. K. Sahu, D. J. Richardson, and D. N. Payne \\ Optoelectronics Research Centre, University of Southampton, Southampton SO17 1BJ, England \\ Phone: +44 (0) 23 8059 3101, fax: +44(0)2380593142 \\ Email:jn@orc.soton.ac.uk \\ * Present address Fianium Ltd., 20 Compass Point, Ensign Way, Southampton SO31 4RA, England
}

\begin{abstract}
High power fiber sources have reached several kilowatts of output power, and are now leading contenders for many applications. Important attractions include control, efficiency, manufacturability, and reliability. We will exemplify opportunities and limitations for these revolutionary sources.

(C)2008 Optical Society of America

OCIS codes: (060.2320) Fiber optics amplifiers and oscillators, (140.3550) Lasers, Raman
\end{abstract}

\section{Introduction}

High power fiber sources are now leading contenders for many important applications requiring powers from a few watts up to several kilowatts. Beyond raw power, fibers offer advantages such as control, efficiency, manufacturability, and reliability. These derive from the fundamental fiber geometry, as well as advances in fiber design, fabrication, and pump diodes, largely driven by the optical communications industry. Master oscillator - power amplifier (MOPA) configurations build on the unique combination of high power, high efficiency, high gain, and broad gain bandwidth of rare-earth (RE) doped fibers. In MOPAs, the output from highly controlled, low power seed lasers can be amplified to ultrahigh power levels whilst preserving the desired seed characteristics. High control is much simpler at low powers. For example, high-speed lithium niobate modulators can be used to control the amplitude as well as the phase of the seed light. This makes MOPAs revolutionary light sources that can enable and dominate a range of devices and application areas. At the same time, the fiber MOPAs are being pushed ever closer to their physical limits. Here, we will exemplify both opportunities and limits of high-power fiber MOPAs.

\section{Fiber Raman amplifier with controllable gain spectrum}

Raman amplifiers offer the potential to generate gain at any arbitrary wavelength over several Stokes orders with an appropriate pump source. This has proved a very effective and successful way of providing gain at wavelengths not directly available with RE-doped fibers. Most of this success has been achieved using CW pump diodes, but in recent years there has been renewed interest in pulsed pumping of Raman amplifiers. This has been mainly in the telecommunications area through time-division multiplexed pumping schemes [1], but also in other areas due to advances in diode-seeded high power fiber MOPA systems [2]. A MOPA allows for excellent control of the pulse parameters which is not easily available from Q-switched or mode-locked lasers. This opens up new opportunities for controlling the Raman gain spectrum through control of the pulse parameters. We have experimentally investigated this, by pumping a fiber Raman amplifier with step-shaped optical pulses delivered from an ytterbium (Yb) doped fiber MOPA [3], [4]. With the instantaneous power of each step appropriately tailored, different parts of the pulse transfer their energy to different Stokes orders, leading to a controllable gain spectrum covering multiple Stokes orders. This opens up opportunities for an ultra-broadband Raman amplifier with near-instantaneous electronic control of the gain spectrum.

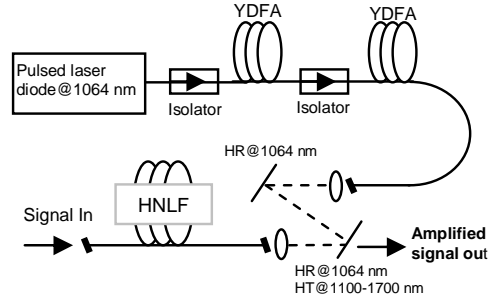

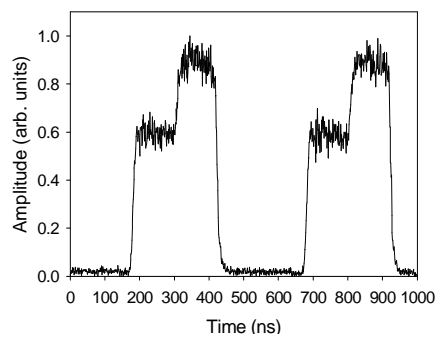

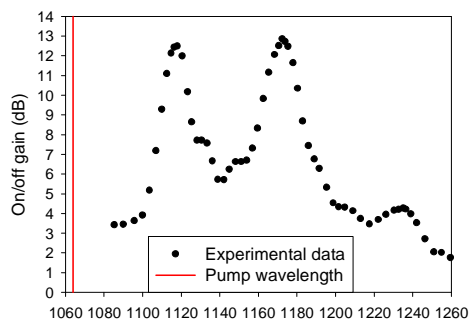

Wavelength $(\mathrm{nm})$

Fig. 1 Fiber Raman amplifier pumped by diode-seeded Yb-doped fiber MOPA with controllable pulse shapes. Experimental set-up, step-shaped pulses, and resulting gain spectrum. YDFA: Yb-doped fiber amplifier, HNLF: highly nonlinear fiber (Sumitomo), HR: high reflectivity, HT: high transmissivity.

In our experiments, we used a directly modulated semiconductor diode at $1064 \mathrm{~nm}$ as the seed laser (Fig. 1) [3], [4]. The diode was controlled by electrical pulses from an arbitrary waveform generator to provide the desired pulse shape. This was then amplified by two cascaded Yb-doped fiber amplifiers (YDFAs) up to $4 \mathrm{~W}$ of average power. The pump light from the was launched into a $2 \mathrm{~km}$ long highly nonlinear fiber fabricated by Sumitomo Electric Industries, Ltd. Figure 1 further shows an example of the pump pulses we used, as well as the resulting 
Raman gain spectrum [4]. The pulses had a total duty cycle of $50 \%$ (25\% for each step). The resulting gain spectrum shows a peak on/off counter-propagating gain of more than $12 \mathrm{~dB}$ over both the $1^{\text {st }}$ and $2^{\text {nd }}$ Stokes orders. We have extended this work to pump pulses with three steps, to create gain over three Stokes orders, and to the use of two seed diodes at different wavelengths, individually shaped in the time domain and amplified by the same YDFAs. These approaches allow us to extend the bandwidth of the Raman gain spectrum and to fill in the gap between the Raman gain peaks in Fig 1. We can also generate higher-order Raman gain without gain at the intermediate orders, and have done so up to the $7^{\text {th }}$ order.

One problem is that Raman amplification is a relatively weak nonlinear process, which requires high intensities over long fiber lengths. Under these conditions, linewidth broadening due to self- and cross-phase modulation can be a problem, especially with pulsed pumping [2]. However, with a fiber MOPA pump source, the narrow seed linewidth that can be obtained with the right seed laser, combined with the temporal control, can help to reduce the linewidth of the output beam. This is illustrated in Fig. 2. To obtain these results experimentally, broadening due to different evolutions of the signal and pump polarizations must be avoided, e.g., through the use of a polarization-maintaining fiber for additional control of the nonlinear interaction.
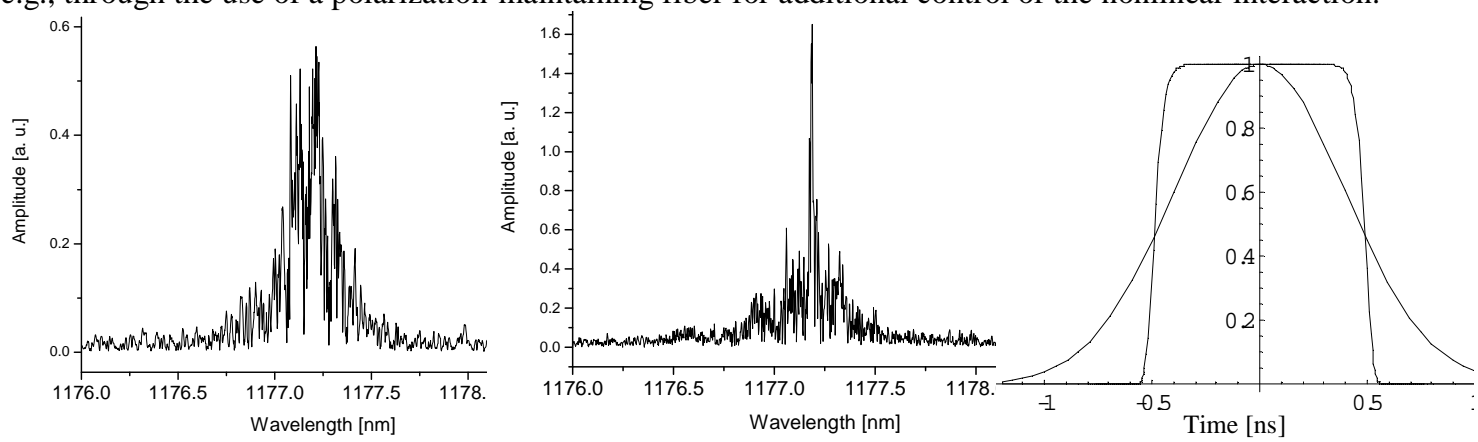

Fig. 2. Simulated spectra at the second Raman Stokes after pulsed co-propagating Raman amplification in a $40 \mathrm{~m}$ long single-mode fiber for different pump pulse shapes. Left: Gaussian pulse. Center: Super-gaussian pulse of order 8. Right: Pump pulse shapes, showing the nearly-rectangular shape of the super-gaussian pulse.

Single-frequency fiber MOPAs and synthetic-aperture lasers

Fiber MOPAs can also provide excellent spectral control. Thus, high-coherence or singlefrequency MOPAs can generate high-power radiation with very narrow linewidths down to the $\mathrm{kHz}$-level. Such MOPAs, operating on a single polarization and a single spatial mode, with linewidths up to the GHz-level, can be coherently combined into a synthetic-aperture laser (Fig. 3). If the phases of individual channels are controlled, a synthetic-aperture laser can be realized with powers that can far exceed that possible from a single fiber.

Stimulated Brillouin scattering (SBS), a nonlinear effect, is a key limitation for highpower fiber amplifiers with the coherence required for synthetic aperture lasers. SBS has

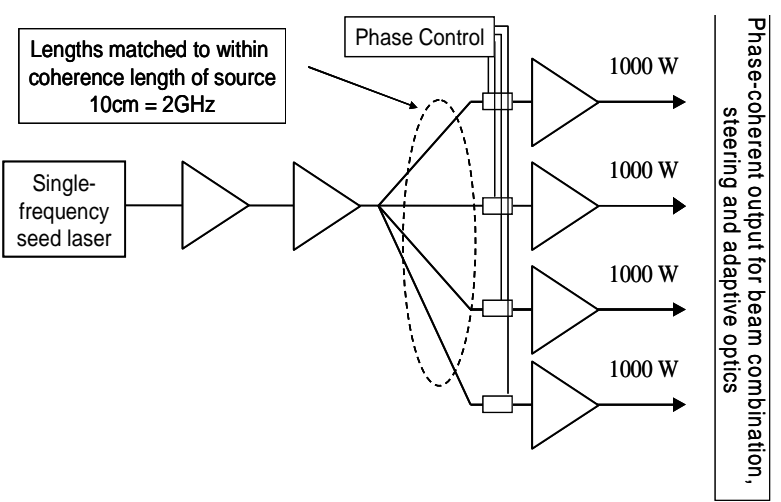

Fig. 3. Synthetic-aperture laser comprising a multi-channel MOPA seeded by a single narrow-line master oscillator. been successfully addressed in recent years, to enable power-scaling to the kilowatt-level of Yb-doped fiber MOPAs (e.g., [5]). One possibility is to rely on the longitudinal temperature variation that is generated in REdoped fibers at high powers [5], [6]. These can reach $100 \mathrm{~K}$ or more. Another possibility is to use a transversally varying speed for the acoustic wave involved in the Brillouin interaction, e.g., with varying germanium and aluminum concentrations across the core [7], [8], [9], [10]. This way, it is possible to independently control propagation of the acoustic and optical waves, and an acoustic speed variation as large as $9 \%$ has been achieved [10]. The resulting SBS suppression is sometimes explained in terms of a reduced overlap with (guided) acoustic modes [9]. However, SBS occurs even in the absence of guided acoustic mode, so this is not always a satisfactory explanation. We have determined the acoustic speed profile (also referred to as the "acoustic refractive index profile") that minimizes the SBS (i.e., the Brillouin gain), based on an approximate expression. We find that the resulting quasi-optimized spectral shape of the Brillouin gain spectrum is the same for all core diameters and (optical) refractive index distributions, and that the magnitude is inversely proportional to the effective area of the (optical) mode. Numerical calculations confirm this, with a high accuracy, in the case of an anti- 
guiding acoustic refractive index profile. The Brillouin gain suppression is $\sim 13 \mathrm{~dB}$, which is larger than the 11.2 $\mathrm{dB}$ reported with triangular antiguiding acoustic refractive index profiles [10], but perhaps not significantly so. If the acoustic refractive index profile is inverted, to form an acoustic waveguide, acoustic modes appear that lead to spikes in the Brillouin gain spectrum. This is a severe effect with small-core fibers, in which the acoustic mode separation significantly exceeds the phonon lifetime-limited Brillouin linewidth of around $50 \mathrm{MHz}$, but only a minor effect with large optical mode areas. See Fig. 4 for a fiber with a $50 \mu \mathrm{m}$ diameter (optical) stepindex core, in which case the appearance of guided acoustic modes increases the peak Brillouin gain by less than $30 \%$. Furthermore, since the peaks are only separated by at most around $100 \mathrm{MHz}$ for a $50 \mu \mathrm{m}$ core, thermal broadening of the Brillouin gain, or even minor broadening of the signal, may well smear out the peaks. Then, there will be almost no difference between the Brillouin gain with a waveguiding and antiguiding acoustic refractive index profiles. We note also that with slight modifications of the acoustic refractive index profile, it should be possible to distribute the acoustic modes more evenly. This would reduce the spectral spacing of the Brillouin peaks, and help to smear them out further (as in the low-frequency end of the spectrum in Fig. 4). Note further that for an unperturbed circular fiber like ours, only circularly symmetric acoustic modes contribute to the Brillouin gain for the fundamental optical mode, which is expected to see the highest gain. Even a relatively small perturbation, such as modest bending of the fiber, will distort the modes, so that a much larger number of acoustic modes will participate in the interaction. Again, this further smears out the Brillouin gain spectrum.

We conclude therefore, that an acoustic antiguide and waveguide, with "opposite" acoustic refractive index profiles, are likely to result in similar Brillouin gain spectra for large-core fibers. Because of the absence of guided acoustic modes, or because of the large number of acoustic modes that are involved in the interaction in large-core fibers, a modal description of the acoustic modes is not necessarily helpful in the understanding of the Brillouin gain with transversally varying acoustic speed profiles. In any case, a modal description cannot treat only a single acoustic mode as sometimes in the past [9], but needs to include all interacting acoustic modes.
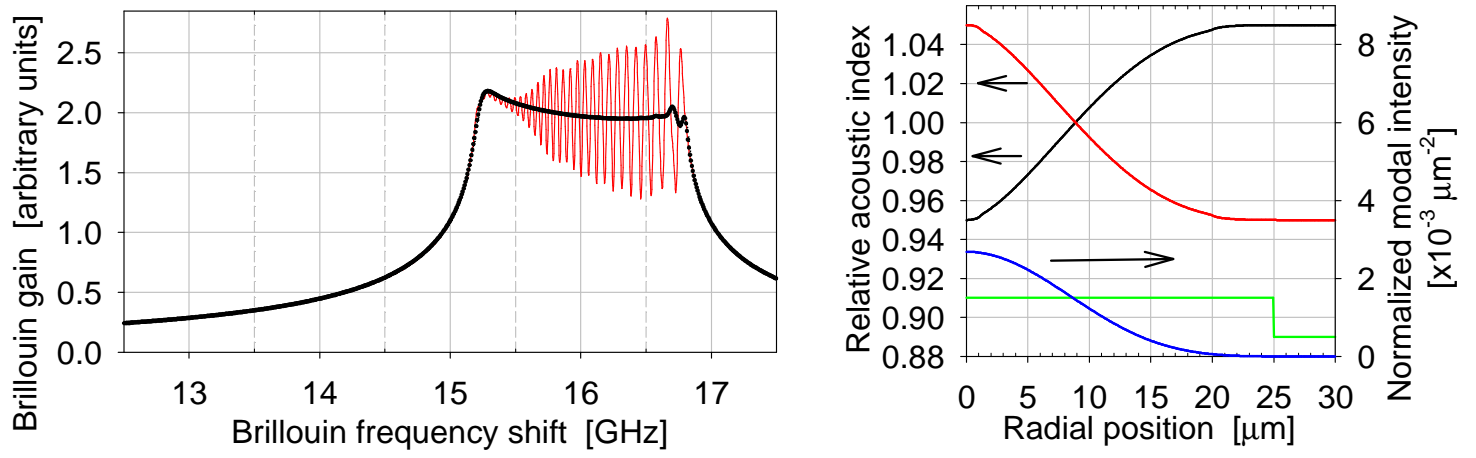

Fig. 4 Spectral shape of Brillouin gain (left) with quasi-optimized antiguiding and guiding acoustic refractive index profiles with 10\% index step (right). The optical step-index core (50 $\mu$ m diameter) and the normalized intensity of the optical mode are indicated, too. Optical wavelength $1080 \mathrm{~nm}$.

In summary, the continuing advances in the control and in the suppression of nonlinearities in high-power fiber sources promises to open up for a large number of exciting new devices and applications.

Acknowledgments: The highly nonlinear fiber was provided by M. Hirano, M. Onishi, and T Sasaki, of Sumitomo Electric Industries. The Raman guidestar work was sponsored by EOARD (grant no. FA8655-04-13065). EPSRC provided student scholarships for P. Dupriez and C. Farrell.

\section{References}

1. L. F. Mollenauer, A. R. Grant, P. V. Mamyshev, "Time-division multiplexing of pump wavelengths to achieve ultrabroadband, flat, backward-pumped Raman gain,” Opt. Lett. 27, 592-594 (2002).

2 P. Dupriez, C. Farrell, M. Ibsen, J. K. Sahu, J. Kim, C. Codemard, Y. Jeong, D. J. Richardson, and J. Nilsson, "1 W average power at 589 nm from a frequency doubled pulsed Raman fiber MOPA system", Proc. SPIE 6102, 61021G (2006)

3 C. Farrell, C. Codemard, J. Nilsson, "A counter-propagating cascaded Raman fiber amplifier pulsed pumped with a $1.06 \mu \mathrm{m}$ source," Frontier in Optics, San Jose, CA 16-20 Sept 2007, FWB2, (2007).

4. C. Farrell, C. Codemard, and J. Nilsson, "A Raman fibre amplifier generating simultaneous gain across multiple Stokes orders by using step shaped optical pulses", Europhoton 2008, Paris France.

5. Y. Jeong, J. Nilsson, J. K. Sahu, D. N. Payne, R. Horley, L. M. B. Hickey, and P. W. Turner, "Power scaling of single-frequency ytterbium-doped fiber master oscillator power amplifier sources up to 500 W", IEEE J. Sel. Top. Quantum Electron. 13, 546-551 (2007)

6. Y. Jeong et al., "Single-frequency single-mode plane-polarized ytterbium-doped fiber master-oscillator power amplifier source with 264 W output power", Opt. Lett. 30, 459-461 (2005)

7. Y. Koyamada, S. Sato, S. Nakamura, H. Sotobayashi, and W. Chujo, "Simulating and designing Brillouin gain spectrum in single mode fibers," J. Lightwave Technol. 22, 631-639 (2004)

8. P. D. Dragic et al., "Optical fiber with an acoustic guiding layer for stimulated Brillouin scattering suppression", CLEO, CThZ3, 2005.

9. M. Li, X. Chen, J. Wang, S. Gray, A. Liu, J. A. Demeritt, A. B. Ruffin, A. M. Crowley, D. T. Walton, and L. A. Zenteno, "Al/Ge codoped large mode area fiber with high SBS threshold", Opt. Express 15, 8290-8299 (2007)

10. M. D. Mermelstein, M. J. Andrejeo, J. Fini, A. Yablon, C. Headley, D. J. DiGiovanni, and A. H. McCurdy, "11.2 dB SBS gain suppression in a large mode area Yb-doped optical fiber", Proc. SPIE 6873, U63-U69 (2008) 